\title{
METRIC PROPERTIES OF POSITIVELY ORDERED MONOIDS.
}

Friedrich WEHRUNG, Université de CAEN, FRANCE

\begin{abstract}
We introduce here an intrinsic (quasi-) metric on each positively ordered monoid (P.O.M.), which is defined in terms of the evaluation map from the given P.O.M. to its bidual and for which P.O.M. homomorphisms are continuous. Moreover, we find a class of refinement P.O.M.'s which, equipped with the canonical metric, are complete metric spaces; this class includes the class of weak cardinal algebras, but also most cases of completions of a certain kind (we will call it 'strongly reduced products') of P.O.M.'s, and of which a prototype has been used in a previous paper for the description of the evaluation map of a given refinement P.O.M.. This result can also be viewed as a wide generalization to the non-linearly ordered case (for example weak cardinal algebras) of the (Cauchy-) completeness of the real line.
\end{abstract}

$\mathcal{A M S}$ subject classification: primary 06F05, 06F30; secondary 28B10. 


\title{
METRIC PROPERTIES OF POSITIVELY ORDERED MONOIDS.
}

\author{
Friedrich WEHRUNG \\ Université de CAEN \\ Département de Mathématiques \\ 14032 CAEN CEDEX, \\ FRANCE.
}

\section{INTRODUCTION; BASIC DEFINITIONS AND NOTATIONS.}

We recall here the definition of positively ordered monoids, as it appears in [15].

Definition. A positively ordered monoid (from now on a P.O.M.) is a structure $(A,+, 0, \leq)$ where $(A,+, 0)$ is a commutative monoid and $\leq$ is a preordering of $A$ such that $(A,+, 0, \leq)$ satisfies the following:

(i) $(\forall a, b, c)(a \leq b \Rightarrow a+c \leq b+c)$,

(ii) $(\forall a)(a \geq 0)$.

In particular, when $(A,+, 0, \leq)$ is a commutative monoid, then we can define a preordering (not always antisymmetric) $\leq$ on $A$ by

$$
(\forall x, y \in A)(x \leq y \Leftrightarrow(\exists z \in A)(x+z=y)) .
$$

Such P.O.M.'s will be called, as in [15], minimal. Tarski's monograph [12] is essentially devoted to the study of certain minimal P.O.M.'s called cardinal algebras, and also to some other weaker but still interesting structures called refinement algebras; it turns out that these algebras appear naturally in very many forms, as for example algebras of isomorphism types of certain structures (as countably complete Boolean algebras), or algebras of positive (possibly infinite) real-valued functions (see [4], [14], [15]), countably distributive lattices, either finite or countable equidecomposability type monoids (see [12], [13]), etc... . Since then, this subject has been studied by several authors (see [4], [5], [8], [9], [10], [11], [15]), and the notion of weak cardinal algebra has been introduced (see [9], [15]). It is very interesting to note that all P.O.M.'s mentioned so far have the so-called finite refinement property, which is the statement

$\left(\forall a_{0}, a_{1}, b_{0}, b_{1}\right)\left(a_{0}+a_{1}=b_{0}+b_{1} \Rightarrow\left(\exists\left(c_{i j}\right)_{i, j<2}\right)(\forall i<2)\left(a_{i}=c_{i 0}+c_{i 1}\right.\right.$ and $\left.\left.b_{i}=c_{0 i}+c_{1 i}\right)\right)$. 
We will say that a P.O.M. is a refinement P.O.M. when it is minimal and satisfies the finite refinement property.

In [15], we try to unify these concepts around an algebraic notion which is exempt, unlike (weak) cardinal algebras, of any presupposed infinite addition; in [15], we chose the notion of injectivity (from the categorical point of view). The fundamental injective object in the category of P.O.M.'s equipped with its natural notion of embedding is the extended positive real line, $\overline{\mathbb{P}}=[0 \infty]$ equipped with its natural notions of addition, zero and ordering (its injectivity is a result of Tarski, and it is not difficult to prove that it is equivalent - in set theory without choice - to the Hahn-Banach extension theorem): every injective P.O.M. embeds into a power of $\overline{\mathbb{P}}$. Thus $\overline{\mathbb{P}}$ is the pivot around which can be built a duality theory of P.O.M.'s, as with $\mathbf{2}$ in the case of Boolean algebras (via Stone's representation theorem), $\mathbb{R} / \mathbb{Z}$ in the case of abelian groups, or $\mathbb{R}$ in the case of normed linear spaces (via the Hahn-Banach extension theorem).

If $A$ and $B$ are two P.O.M.'s, then $\operatorname{Hom}(A, B)$ denotes the P.O.M. of all homomorphisms from $A$ to $B$ (for both the monoid structure and the preordered set structure), equipped with its componentwise preordering. If $A$ is a P.O.M., then the dual of $A$ is $A^{*}=\operatorname{Hom}(A, \overline{\mathbb{P}})$, and the natural evaluation map $e_{A}: x \mapsto x^{* *}$ from $A$ to its bidual $A^{* *}$, which is an embedding if and only if $A$ embeds into an injective P.O.M. - (we will say that $A$ is regular) and this last possibility does not always occur. K.P.S. Bhaskara Rao and R.M. Shortt proved in [9] that if $\left(A,+, 0, \sum\right)$ is a weak cardinal algebra, then $(A,+, 0, \leq)(\leq$ minimal) is a regular P.O.M., while in [15], we described in a convenient way the evaluation map of any refinement P.O.M., which implied easily the previous result.

Unfortunately, $A^{* *}$ is in general a very unwieldy object (essentially too big), and so is $e_{A}$; for example, if $A$ is a cardinal algebra, $e_{A}$ is not in general countably additive for the natural structure of cardinal algebra on both sides (see [11]). In this paper, we will show some positive results of preservation of properties of countable character by $e_{A}$. In fact, $e_{A}$ will yield us a natural, intrinsic quasi-metric $\delta$ on $A$, which will be a metric if $e_{A}$ is an embedding (throughout this paper, a quasi-distance is allowed to take infinite values) see paragraph 1. It will allow us to construct some new regular (see 2.1 and 2.2) P.O.M.'s, which are generalisations of the $\mathrm{K}(A)$ introduced in [15], which we will call strongly reduced products. These, in turn, will be very useful by giving us some new information about the map $e_{A}$ mentioned above (see 2.3 and the example following). Also, the natural metric will turn out to be complete in several natural cases (see 4.7), including weak cardinal algebras and some of the strongly reduced products mentioned above (including the $\mathrm{K}(A)$ 's). This last result seems to us to be especially worth mentioning, because there are actually very 
few examples where some algebraic (most often ordered) structure carries an intrinsic metric which turns out to be complete. Moreover, our metric seems to have nothing to do with (complete or not) lattices, where it trivializes; it rather seems to generalize to a large number of cases (including weak cardinal algebras) where the interval topology is not suitable for study (the ordering is not linear) the well-known and essentially isolated fact that the real line $\mathbb{R}$, equipped with its intrinsic uniform (metrizable) structure, is complete. It seems in fact that those spaces which we consider can be rather often used as image spaces of finitely additive measures, and thus, in order to satisfy non trivial properties of infinite character, they must satisfy some completeness properties, of which metric completeness might be only one particular aspect. See e.g. [10] for more precise details about this.

In this paper, we will use basic set-theoretical notation and terminology; if $f$ is a map from a set $X$ to a set $Y$ and $Z$ is a subset of $X$, then $f Z$ will denote the image of $Z$ under $f$; if $Z$ is a subset of $Y$, then $f^{-1} Z$ will denote the inverse image of $Z$ under $f ; X^{Y}$ will denote the set of all maps from $Y$ to $X$. Families will be denoted by $\left(a_{i}\right)_{i \in I}$, or $\left(a_{i}\right)_{i}$ when there is no ambiguity about the index set. The set of all natural numbers will be denoted by $\omega$ or by $\mathbb{N}$ whether it will be considered as an ordinal or as, say, a P.O.M.. If $\mathcal{F}$ is a filter on some set $I$ and $\varphi(x)$ is some statement with $x$ as free variable, then the statement $\left(\forall^{\mathcal{F}} x\right) \varphi(x)$ means that $\{x \in I: \varphi(x)\} \in \mathcal{F}$. In the case where $\mathcal{F}$ is the Fréchet filter over $\omega$, we will write $\forall^{\infty}$ for $\forall^{\mathcal{F}}$. If $X$ and $Y$ are two preordered sets, then a map $f$ from $X$ to $Y$ is increasing (resp. decreasing) when for all $x, y$ in $X$ such that $x \leq y$, we have $f(x) \leq f(y)$ (resp. $f(y) \leq f(x)$ ). A preordered set $X$ is left (resp. right) directed when any two elements of $X$ have a lower (resp. upper) bound. If $X$ and $Y$ are two subsets of some preordered set $P$, then we write $X \leq Y$ for $(\forall(x, y) \in X \times Y)(x \leq y)$; when $X$ (resp. $Y$ ) is a singleton, $\{a\}$, we write $a \leq Y$ (resp. $X \leq a$ ). If $\alpha$ and $\beta$ are two cardinals, then $P$ satisfies the $(\alpha, \beta)$-interpolation property when for any nonempty sets $X$ and $Y$ of size respectively $\leq \alpha$ and $\leq \beta$ such that $X \leq Y$, there is $a$ in $P$ such that $X \leq a \leq Y$; if $X$ is a subset of $P$, then we denote by $\bigwedge X$ (resp. $\bigvee X)$ the g.l.b. (resp. the l.u.b.) of $X$ when it exists. Furthermore, suppose that $P=\overline{\mathbb{P}}$, let $\mathcal{F}$ be a filter on a set $I$ and let $\left(a_{i}\right)_{i \in I}$ be a $I$-indexed family of elements of $\overline{\mathbb{P}}$; we define $\overline{\lim }_{\mathcal{F}}\left(a_{i}\right)_{i \in I}=\bigwedge_{X \in \mathcal{F}} \bigvee_{i \in X} a_{i}$ and $\underline{\lim }_{\mathcal{F}}\left(a_{i}\right)_{i \in I}=\bigvee_{X \in \mathcal{F}} \bigwedge_{i \in X} a_{i}$; when both are equal, we shall denote them by $\lim _{\mathcal{F}}\left(a_{i}\right)_{i \in I}$ (it is the usual notion of limit for $\overline{\mathbb{P}}$ equipped with its natural topology). Furthermore, we shall drop the index set $I$ in the notations above where there is no ambiguity.

If $E$ is a set and $d$ is a map from $E \times E$ to $\overline{\mathbb{P}}$, we shall say that $d$ is a quasi-distance 
when for all $x, y, z$ in $E$, we have:

$$
d(x, x)=0 ; d(x, y)=d(y, x) ; d(x, z) \leq d(x, y)+d(y, z) .
$$

Furthermore, if we have in addition $(\forall x, y \in E)(d(x, y)=0 \Rightarrow x=y)$, then we will say that $d$ is a distance. The only difference between the notions of quasi-distance or distance presented here and the usual notions lies in the fact that (quasi-) distances may assume infinite values - since the quasi-distance considered in this paper has a simple 'concrete' signification, we chose not to alter it by replacing it by some uniformly equivalent bounded distance.

Let us now give some more definitions about P.O.M.'s (some of which are already known).

If $a$ and $b$ are in some P.O.M., we say that $a \ll b$ when $a+b=b$, and that $a$ is idem-multiple when $2 a=a$. The pseudo-cancellation property (see [15]) is the statement

$$
(\forall a, b, c)(a+c \leq b+c \Rightarrow(\exists e \ll c)(a \ll b+e)) .
$$

A strong refinement P.O.M. is an antisymmetric refinement P.O.M. satisfying the pseudo-cancellation property (see [15]). If $\kappa$ is a cardinal, then $A$ satisfies the $\kappa$-absorption property when

$$
\left(\forall\left(a_{i}\right)_{i} \in A^{\kappa}\right)(\forall b \in A)\left((\forall i<\kappa)\left(a_{i} \ll b\right) \Rightarrow(\exists a \ll b)(\forall i<\kappa)\left(a_{i} \leq a\right)\right) .
$$

Say that a P.O.M. is Archimedean (see also [15]) when it satisfies the statement

$$
(\forall a, b)((\forall n \in \mathbb{N})(n a \leq b) \Rightarrow a \ll b)
$$

Finally, if $m \in \mathbb{N} \backslash\{0\}$, say that the multiplicative $m$ - $\leq$-cancellation property is the statement

$$
(\forall x, y)(m x \leq m y \Rightarrow x \leq y)
$$

and the multiplicative $\leq$-cancellation property is the conjunction of the multiplicative $m$ - $\leq$-cancellation property for all $m$ in $\mathbb{N} \backslash\{0\}$.

Finally, we denote by $\left(x \mapsto e^{x}\right)$ the exponential function on $\overline{\mathbb{P}}$ (with $e^{\infty}=\infty$ ). 


\section{THE CANONICAL QUASI-DISTANCE.}

Definition. Let $A$ be a P.O.M.. For all $a, b$ in $A$, we put

$$
\rho_{A}(a, b)=\bigwedge\left\{r \in \mathbb{R}_{+}: a^{* *} \leq e^{r} b^{* *}\right\},
$$

and

$$
\delta_{A}(a, b)=\rho_{A}(a, b) \vee \rho_{A}(b, a) .
$$

(As usual, we make the convention $\bigwedge \emptyset=\infty$ ).

Lemma 1.1. Let $A$ be a P.O.M., let $a, b, c, a^{\prime}, b^{\prime}$ in $A$. Then we have

(i) $\rho_{A}(a, a)=\delta_{A}(a, a)=0$,

(ii) $\delta_{A}(a, b)=\delta_{A}(b, a)$,

(iii) $\rho_{A}(a, c) \leq \rho_{A}(a, b)+\rho_{A}(b, c)$,

(iv) $\delta_{A}(a, c) \leq \delta_{A}(a, b)+\delta_{A}(b, c)$,

(v) $\rho_{A}\left(a^{\prime}, b^{\prime}\right) \leq \rho_{A}(a, b)+\delta_{A}\left(a, a^{\prime}\right)+\delta_{A}\left(b, b^{\prime}\right)$,

(vi) $\rho_{A}\left(a+b, a^{\prime}+b^{\prime}\right) \leq \rho_{A}\left(a, a^{\prime}\right) \vee \rho_{A}\left(b, b^{\prime}\right)$,

(vii) $\delta_{A}\left(a+b, a^{\prime}+b^{\prime}\right) \leq \delta_{A}\left(a, a^{\prime}\right) \vee \delta_{A}\left(b, b^{\prime}\right)$,

(viii) $\rho_{A}(a, b)=0 \Leftrightarrow a^{* *} \leq b^{* *}$,

(ix) $\delta_{A}(a, b)=0 \Leftrightarrow a^{* *}=b^{* *}$.

Proof. A simple verification.

Note that $\rho_{A}$ and $\delta_{A}$ trivialize on the set of idem-multiple elements of $A$. Thus, we may say that their study is relevant only on those P.O.M.'s with non idem-multiple elements.

It follows immediately that $\delta_{A}$ is a quasi-distance on $A$, which we will call the canonical quasi-distance on $A$, and that if $A$ is regular, then $\delta_{A}$ is a distance. As the following example shows, the converse of the latter statement is false.

Example 1. Let $\mathbb{R}_{+}=\{x \in \mathbb{R}: x \geq 0\}$, and let $A$ be the submonoid of $\mathbb{R}_{+} \times \mathbb{R}_{+}$ defined by

$$
A=\left\{(x, y) \in \mathbb{R}_{+} \times \mathbb{R}_{+}: y>0\right\} \cup\{(0,0)\} .
$$

We equip $A$ with its minimal ordering $\leq_{A}$ (note that $\leq_{A}$ is strictly contained in the ordering induced by the natural ordering of $\mathbb{R}_{+} \times \mathbb{R}_{+}$). Let $p$ and $q$ be the projections from $A$ on respectively the first and the second coordinate. If $a$ and $b$ are any two elements 
of $A$ such that $a^{* *}=b^{* *}$, then $p(a)=p(b)$ and $q(a)=q(b)$, whence $a=b$. Thus $\delta_{A}$ is a distance. On the other hand, let $a=(1,1)$ and $b=(2,1)$. Then $a \not_{A} b$, but for all $n$ in $\mathbb{N}$, we have $n a \leq_{A}(n+1) b$ whence $a^{* *} \leq b^{* *}$. Thus $A$ is not regular.

Note that this example satisfies the finite refinement property. One can show with more work that there such an example realized as some submonoid of the set $\mathbb{Q}_{+}$of all positive rationals, equipped with its minimal ordering.

Proposition 1.2. Let $A, B$ be two P.O.M.'s, let $f$ in $\operatorname{Hom}(A, B)$. Then for all $a, b$ in $A$, we have

$$
\rho_{B}(f(a), f(b)) \leq \rho_{A}(a, b) \text { and } \delta_{B}(f(a), f(b)) \leq \delta_{A}(a, b)
$$

Moreover, if $f^{* *}$ is an embedding from $A^{* *}$ into $B^{* *}$, then the inequality above can be replaced by an equality.

Proof. We just show the proof for $\rho_{A}$. Let $r=\rho_{A}(a, b)$. The first inequality is trivial if $r=\infty$; if $r<\infty$, then we have $a^{* *} \leq e^{r} b^{* *}$, thus

$$
f(a)^{* *}=f^{* *}\left(a^{* *}\right) \leq f^{* *}\left(e^{r} b^{* *}\right)=e^{r} f^{* *}\left(b^{* *}\right)=e^{r} f(b)^{* *},
$$

so that the first inequality follows. Now let us suppose that $f^{* *}$ is an embedding; put $r^{\prime}=\rho_{B}(f(a), f(b))$; to conclude, it suffices to prove that $r \leq r^{\prime}$. It is true if $r^{\prime}=\infty$; otherwise, we have $f(a)^{* *} \leq e^{r^{\prime}} f(b)^{* *}$, thus $f^{* *}\left(a^{* *}\right) \leq f^{* *}\left(e^{r^{\prime}} b^{* *}\right)$, hence $a^{* *} \leq e^{r^{\prime}} b^{* *}$; hence $r \leq r^{\prime}$, and the conclusion follows.

It follows that P.O.M. homomorphisms are continuous with respect to the quasidistance topology induced by $\delta$.

Corollary 1.3. $\rho$ and $\delta$ are intrinsic, which means that if $A$ is a sub-P.O.M. of a P.O.M. $B$ and if $a, b$ are in $A$, then we have

$$
\rho_{A}(a, b)=\rho_{B}(a, b) \text { and } \delta_{A}(a, b)=\delta_{B}(a, b) .
$$

Consequently, we will just write $\rho(a, b)(\operatorname{resp} . \delta(a, b))$ instead of $\rho_{A}(a, b)\left(\operatorname{resp} . \delta_{A}(a, b)\right)$.

Tarski was the first one to notice the injectivity of $\overline{\mathbb{P}}$ (see [13]); in fact, his proof applies to the following lemma, which we will thus state without proof (and which is another way to prove 1.3): 
Lemma 1.4. Let $A$ be a P.O.M., let $r$ in $\overline{\mathbb{P}}$, let $a, b$ in $A$. Then the following are equivalent:

(i) $a^{* *} \leq r . b^{* *}$,

(ii) $(\forall \varepsilon>0)(\exists p, q \in \mathbb{N} \backslash\{0\})(p / q \leq r+\varepsilon$ and $q a \leq p b)$.

\section{STRONGLY REDUCED PRODUCTS OF P.O.M.'s.}

Let $\left(A_{i}\right)_{i \in I}$ be a family of P.O.M.'s, let $\mathcal{F}$ be a [proper] filter over $I$. We equip the product $\prod_{i \in I} A_{i}$ with componentwise addition and the binary relations respectively defined by

$$
\left(a_{i}\right)_{i} \equiv\left(b_{i}\right)_{i} \Leftrightarrow \lim _{\mathcal{F}} \delta\left(a_{i}, b_{i}\right)=0
$$

and

$$
\left(a_{i}\right)_{i} \leq\left(b_{i}\right)_{i} \Leftrightarrow \lim _{\mathcal{F}} \rho\left(a_{i}, b_{i}\right)=0 .
$$

Using 1.1 , it is immediate to see that $\equiv$ is an equivalence on $\prod_{i \in I} A_{i}$, compatible with the addition and that $\leq$ is a preordering containing $\equiv$. The quotient structure of $\prod_{i \in I}\left(A_{i},+, 0, \leq\right)$ by $\equiv$ is obviously a P.O.M., which we will denote by $\bar{\prod}_{\mathcal{F}}\left(A_{i}\right)_{i \in I}$, or just $\bar{\prod}_{\mathcal{F}} A_{i}$, and we will call it the strongly reduced product of $\left(A_{i}\right)_{i} \operatorname{modulo} \mathcal{F}$. If $a=\left(a_{i}\right)_{i}$ is an element of $\prod_{i \in I} A_{i}$, then we will denote its equivalence class modulo $\equiv$ by $[a]=\left[a_{i}\right]_{i}$. Obviously, $\bar{\Pi}_{\mathcal{F}} A_{i}$ is a quotient structure of the usual reduced product of $\left(A_{i}\right)_{i \in I}$ by $\mathcal{F}$ (see [3], chapter 4 for basic facts about reduced products).

The following theorem gives a convenient expression of $\rho$ and $\delta$ in a strongly reduced product.

Theorem 2.1. Let $\left(A_{i}\right)_{i \in I}$ be a family of P.O.M.'s, let $\mathcal{F}$ be a filter over $I$, let $a=\left[a_{i}\right]_{i}$ and $b=\left[b_{i}\right]_{i}$ in $\bar{\prod}_{\mathcal{F}} A_{i}$. Then we have

$$
\rho(a, b)=\varlimsup_{\mathcal{F}} \rho\left(a_{i}, b_{i}\right)
$$

and

$$
\delta(a, b)=\varlimsup_{\mathcal{F}} \delta\left(a_{i}, b_{i}\right)
$$

Proof. Since $\varlimsup$ im commutes with $\vee$, it suffices to prove the equality for $\rho$. Put $A=\bar{\prod}_{\mathcal{F}} A_{i}, r=\rho(a, b), r_{i}=\rho\left(a_{i}, b_{i}\right), \bar{r}=\overline{\lim }_{\mathcal{F}} r_{i}$. We first prove that $\bar{r} \leq r$; if $r=\infty$ 
then this is obvious, so suppose that $r<\infty$. We have $a^{* *} \leq e^{r} b^{* *}$ in $A^{* *}$, thus, using 1.4, for all $\varepsilon>0$ there are $m, n$ in $\mathbb{N}$ such that $0<m<\left(e^{r}+\varepsilon\right) n$ and $n a \leq m b$. Let $\eta>0$ such that $\left(e^{\eta}-1\right) m \leq \varepsilon n$. By definition of the canonical preordering of $A$, there is $X$ in $\mathcal{F}$ such that $(\forall i \in X)\left(\rho\left(n a_{i}, m b_{i}\right) \leq \eta\right)$. So for all $i$ in $X, A_{i}^{* *}$ satisfies $n a_{i}^{* *} \leq e^{\eta} m b_{i}^{* *}$, thus $n a_{i}^{* *} \leq(m+\varepsilon n) b_{i}^{* *} \leq n\left(e^{r}+2 \varepsilon\right) b_{i}^{* *}$, so that $\rho\left(a_{i}, b_{i}\right) \leq \ln \left(e^{r}+2 \varepsilon\right) \leq r+2 \varepsilon$; thus $\bar{r} \leq r+2 \varepsilon$. Letting $\varepsilon$ evaporate yields $\bar{r} \leq r$.

Conversely, let $X$ in $\mathcal{F}$; put $r_{X}=\bigvee_{i \in X} r_{i}$, we will prove that $r \leq r_{X}$; if $r_{X}=\infty$ then this is trivial, so suppose that $r_{X}<\infty$. Fix $p, q$ in $\mathbb{N} \backslash\{0\}$ such that $e^{r_{X}} \leq p / q$. For all $i$ in $X$, we have $a_{i}^{* *} \leq e^{r_{X}} b_{i}^{* *}$, thus $q a_{i}^{* *} \leq p b_{i}^{* *}$, thus $\rho\left(q a_{i}, p b_{i}\right)=0$; this holds for all $i$ in $X$, thus $q a \leq p b$, thus $a^{* *} \leq(p / q) b^{* *}$. This holds for all possible values of $p, q$, thus $a^{* *} \leq e^{r_{X}} b^{* *}$; hence, $r \leq r_{X}$. This holds for all $X$ in $\mathcal{F}$, thus $r \leq \bar{r}$. The conclusion follows.

COROLlary 2.2. Every strongly reduced product of P.O.M.'s is a regular P.O.M..

Proof. Obvious from 2.1 and the definition of the preordering of a strongly reduced product.

As an application, we will get a better understanding of the evaluation map of any P.O.M.. If $A$ is a P.O.M. and $X$ is a subset of $A$, we will denote by $\wedge^{*} X$ (resp. $\bigvee^{*} X$ ) the g.l.b. (resp. the l.u.b.) of $e_{A} X$ in $\overline{\mathbb{P}}^{A^{*}}$; so if $X$ is left (resp. right) directed, then it is the same to take the g.l.b. (resp. the l.u.b.) of $e_{A} X$ in $A^{* *}$. Moreover, we will write $X^{+}$ (resp. $X^{-}$) the set of majorants (resp. minorants) of all elements of $X$ in $P$.

Proposition 2.3. Let $A$ be a P.O.M., let $X, Y$ be two nonempty subsets of $A$. Then the following are equivalent:

(i) $\bigwedge^{*} X \leq \bigvee^{*} Y$

(ii) For every homomorphism from $A$ to a regular P.O.M. E, we have $(f X)^{-} \leq(f Y)^{+}$.

Moreover, if $e_{A} X$ (resp. $e_{A} Y$ ) is left-directed (resp. right-directed), then each of the conditions (i), (ii) is equivalent to the following third condition:

(iii) $(\forall \varepsilon>0)(\exists(x, y) \in X \times Y)(\rho(x, y) \leq \varepsilon)$.

Proof. (ii) $\Rightarrow$ (i) is obvious (take $E=\overline{\mathbb{P}}^{A^{*}}, f=e_{A}$ ). Now, assume (i), and let $f$ be in $\operatorname{Hom}(A, E)$ with $E$ regular, let $a$ in $(f X)^{-}, b$ in $(f Y)^{+}$. To prove that $a \leq b$, it is sufficient, since $E$ is regular, to prove that for all $u$ in $E^{*}$, we have $u(a) \leq u(b)$. Let $v=u \circ f$. By hypothesis (i), $\overline{\mathbb{P}}$ satisfies $\bigwedge_{x \in X} v(x) \leq \bigvee_{y \in Y} v(y)$. Hence, we have 


$$
\begin{aligned}
u(a) & \left.\leq \bigwedge_{x \in X} u \circ f(x) \text { (because } a \in(f X)^{-} \text {in } E\right) \\
& =\bigwedge_{x \in X} v(x) \\
& \leq \bigvee_{y \in Y} v(y) \\
& =\bigvee_{y \in Y} u \circ f(y) \\
& \left.\leq u(b) \text { (because } b \in(f Y)^{+} \text {in } E\right),
\end{aligned}
$$

which proves (ii). Hence (i) $\Leftrightarrow($ ii).

Now, put $e=e_{A}$ and suppose that $e X$ and $e Y$ are respectively left-directed and rightdirected. It is easy to see that (iii) implies (i) (we do not use the directedness hypothesis). Conversely, assume (ii). Let $I=e X \times e Y$, equipped with the filter $\mathcal{F}$ generated by the sets $F_{x, y}=\left\{\left(x^{\prime}, y^{\prime}\right) \in I: x^{\prime} \leq x\right.$ and $\left.y \leq y^{\prime}\right\}$ for $(x, y) \in I$. Then $\bar{A}=\bar{\prod}_{\mathcal{F}} e A$ is, by 2.1 , a regular P.O.M.. Let $f$ be the natural map from $A$ to $\bar{A}$. Thus $(f X)^{-} \leq(f Y)^{+}$. But if we put $a=[x:(x, y) \in I]$ and $b=[y:(x, y) \in I]$, then it is easy to see that $a \in(f X)^{-}$and $b \in(f Y)^{+}$; it follows that $a \leq b$, which means by definition that $\lim _{\mathcal{F}} \rho(x, y)=0$, which implies immediately our conclusion.

For example, if $a, a_{n}(n \in \omega)$ are elements of some P.O.M. $A$ with $\left(a_{n}\right)_{n}$ increasing, then $a \leq \bigvee_{n}^{*} a_{n}$ if and only if $(\forall \varepsilon>0)(\exists n \in \omega)\left(\rho\left(a, a_{n}\right) \leq \varepsilon\right)$. In particular, if $a, b$ are in $A$, then $a \leq \infty b$ (in $\left.A^{* *}\right)$ if and only if $(\exists n \in \mathbb{N})(a \leq n b)$. (We say that in this case, $a$ is $b$-bounded).

Another remarkable property of strongly reduced products is a countable saturationlike property. If $s$ and $t$ are two terms of the first-order language $(+, 0)$ and $\varepsilon>0$, then we define the $\varepsilon$-approximations of atomic formulas by

$$
\left.(s=t)\right|_{\varepsilon} \text { is } \delta(s, t) \leq \varepsilon,
$$

and

$$
\left.(s \leq t)\right|_{\varepsilon} \text { is } \rho(s, t) \leq \varepsilon .
$$

If $\vec{a}=\left(\vec{a}^{n}\right)_{n \in \omega}$ with $\vec{a}^{n}=\left(a_{i}^{n}\right)_{i \in I}$, we put $\vec{a}_{i}=\left(a_{i}^{n}\right)_{n}$; moreover, if [-] is an operation defined on $I$-sequences, then we write $[\vec{a}]=\left(\left[\vec{a}^{n}\right]\right)_{n}$. Finally, a filter $\mathcal{F}$ will be said to be anticomplete when it satisfies the following statement: 


$$
\left(\exists\left(X_{n}\right)_{n \in \omega} \in \mathcal{F}^{\omega}\right)\left(\bigcap_{n} X_{n}=\emptyset\right)
$$

With these conventions, we can state in a convenient way the following

Proposition 2.4. Let $\mathcal{F}$ be an anticomplete filter over a set $I$, let $\left(A_{i}\right)_{i \in I}$ be a family of P.O.M.'s; put $A=\prod_{i} A_{i}$. Let $\left(\varphi_{k}(\vec{x}, \vec{y})\right)_{k \in \omega}$ be a countable list of atomic formulas of the language $(+, 0, \leq)$, let $\vec{a}=\left(\vec{a}^{n}\right)_{n}$ in $\left(A^{\omega}\right)^{\omega}$. Put $\bar{A}=\bar{\prod}_{\mathcal{F}} A_{i}$. Then the following are equivalent:

(i) $\left(\exists \vec{x} \in\left(A^{\omega}\right)^{\omega}\right)(\forall k<\omega)\left(\vec{A} \models \varphi_{k}([\vec{x}],[\vec{a}])\right)$;

(ii) $(\forall n \in \omega)\left(\exists \vec{x} \in\left(A^{\omega}\right)^{\omega}\right)(\forall k<n)\left(\vec{A} \models \varphi_{k}([\vec{x}],[\vec{a}])\right)$;

(iii) $(\forall n \in \omega \backslash\{0\})\left(\exists \vec{x} \in\left(A^{\omega}\right)^{\omega}\right)(\forall k<n)\left(\forall^{\mathcal{F}} i\right)\left(\left.A_{i} \models \varphi_{k}\right|_{\frac{1}{n}}\left(\vec{x}_{i}, \vec{a}_{i}\right)\right)$.

Heuristically, the statement of proposition 2.4 says that a countable type of atomic formulas is satisfiable in $\bar{\Pi}_{\mathcal{F}} A_{i}$ if and only if it is 'finitely approximately satisfiable'.

Proof. $\quad(\mathrm{i}) \Rightarrow($ ii) and (ii) $\Rightarrow$ (iii) are trivial. To prove (iii) $\Rightarrow(\mathrm{i})$, we use a diagonal argument. So assume (iii). Using anticompleteness of $\mathcal{F}$, we get a decreasing sequence $\left(X_{n}\right)_{n}$ of elements of $\mathcal{F}$ and a sequence $\left(\vec{x}^{n}\right)_{n \in \omega}$ of elements of $\left(A^{\omega}\right)^{\omega}$ such that $\bigcap_{n} X_{n}=\emptyset$ and

$$
(\forall n \in \omega \backslash\{0\})(\forall k<n)\left(\forall i \in X_{n}\right)\left(\left.A_{i} \models \varphi_{k}\right|_{\frac{1}{n}}\left(\left(\vec{x}^{n}\right)_{i}, \vec{a}_{i}\right)\right) .
$$

Let $\left(h: X_{0} \rightarrow \omega, i \mapsto\right.$ largest $n$ in $\omega$ such that $\left.i \in X_{n}\right)$. Since $\bigcap_{n} X_{n}=\emptyset, h$ is everywhere defined on $X_{0}$. Define now $\vec{x}$ in $\left(A^{\omega}\right)^{\omega}$ by putting $\vec{x}_{i}=\left(\vec{x}^{h(i)}\right)_{i}$ when $i \in X_{0}$, 0 otherwise. Let $k$ in $\omega$, let us prove that $\bar{A} \models \varphi_{k}([\vec{x}],[\vec{a}])$. By definition of equality and preordering of $\bar{A}$, it is sufficient to prove that for all $n>k$ in $\omega$, we have

$$
\left(\forall^{\mathcal{F}} i\right)\left(\left.A_{i} \models \varphi_{k}\right|_{\frac{1}{n}}\left(\vec{x}_{i}, \vec{a}_{i}\right)\right) .
$$

Let $i$ in $X_{n}$. Then $k<n \leq h(i)$ and $i \in X_{h(i)}$, thus, by $(1),\left.A_{i} \models \varphi_{k}\right|_{\frac{1}{h(i)}}\left(\left(\vec{x}^{h(i)}\right)_{i}, \vec{a}_{i}\right)$. But $1 / h(i) \leq 1 / n$, thus we also have $\left.A_{i} \models \varphi_{k}\right|_{\frac{1}{n}}\left(\left(\vec{x}^{h(i)}\right)_{i}, \vec{a}_{i}\right)$, which means that $A_{i} \models$ $\left.\varphi_{k}\right|_{\frac{1}{n}}\left(\vec{x}_{i}, \vec{a}_{i}\right)$. This proves (2), and completes the proof.

Note that when $\mathcal{F}$ is not anticomplete, $\bar{\Pi}_{\mathcal{F}} \mathbb{Q}_{+}$does not satisfy the saturation property above. 


\section{CASE OF REFINEMENT P.O.M.'s.}

In this paragraph, we will need the results of the last chapter of [15], essentially the 'first embedding theorem', which will allow us to redefine the intrinsic quasi-metric structure on refinement P.O.M.'s in a more convenient way. We will just recall without proof the essential construction. Let $A$ be a refinement P.O.M.. If $a, b$ are in $A$ and $n$ in $\mathbb{N}$, then we define the following relations:

$$
\begin{gathered}
a \ll_{n} b \Leftrightarrow(\exists m \in \mathbb{N})\left(2^{m+n} a \leq 2^{m} b\right), \\
a \leq_{n} b \Leftrightarrow\left(\exists c \ll_{n} b\right)(a \leq b+c),
\end{gathered}
$$

and

$$
a \equiv_{n} b \Leftrightarrow\left(\exists u \ll_{n} a\right)\left(\exists v \ll_{n} b\right)(a+u=b+v)
$$

Then we define two binary relations $\equiv$ and $\leq$ on $A^{\omega}$ by

$$
\left(a_{i}\right)_{i} \equiv\left(b_{i}\right)_{i} \Leftrightarrow(\forall m \in \mathbb{N})\left(\forall^{\infty} i\right)\left(a_{i} \equiv_{m} b_{i}\right)
$$

and

$$
\left(a_{i}\right)_{i} \leq\left(b_{i}\right)_{i} \Leftrightarrow(\forall m \in \mathbb{N})\left(\forall^{\infty} i\right)\left(a_{i} \leq_{m} b_{i}\right)
$$

As in the former case of strongly reduced products, the quotient $A^{\omega} / \equiv$ can be equipped with a natural structure of P.O.M., which we will denote by $\mathrm{K}(A)$. One can easily verify that $\mathrm{K}$ is a functor.

Heuristically, [the natural map from $A$ to] $\mathrm{K}(A)$ has been introduced on purpose to be able to define a 'limit' of every sequence $\left(a_{n}\right)_{n}$ of elements of $A$, namely by putting $\operatorname{Lim}_{n \rightarrow \infty} a_{n}=\left[a_{n}\right]_{n}$, such that furthermore, this notion of limit is a homomorphism for the monoid structure and both binary relations $\equiv$ and $\leq$ defined above; in fact, by definition, $\mathrm{K}(A)$ is universal with this property.

In [15] (first embedding theorem), we have proved several properties of $\mathrm{K}(A)$ implying that $\mathrm{K}(A)$ is a regular P.O.M. With the help of proposition 2.3, it is then easy to get the following 
Lemma 3.1. Let $A$ be a refinement P.O.M., let $a, b$ in $A, n$ in $\mathbb{N}$. Then the following holds:

(i) $a \leq_{n} b$ implies $\rho(a, b) \leq \ln \left(1+2^{-n}\right)$;

(ii) If $n \geq 2$, then $\rho(a, b) \leq \ln \left(1+2^{-n}\right)$ implies $a \leq_{n-2} b$.

Proof. Assume first (i). If $a \leq_{n} b$, then $a^{* *} \leq\left(1+2^{-n}\right) b^{* *}$, thus $\rho(a, b) \leq \ln \left(1+2^{-n}\right)$. Conversely, if $\rho(a, b) \leq \ln \left(1+2^{-n}\right)$ holds, then we have $2^{n} a^{* *} \leq\left(2^{n}+1\right) b^{* *}$. Let $\epsilon$ be the canonical homomorphism from $A$ to $\mathrm{K}(A)$. Since $\mathrm{K}(A)$ is regular, it follows from 2.3 that $2^{n} \epsilon(a) \leq\left(2^{n}+1\right) \epsilon(b)$; since $\mathrm{K}(A)$ is a strong refinement P.O.M. satisfying the multiplicative $\leq$-cancellation property, it follows from results in chapter 6 of [15] that there is $\gamma$ in $\mathrm{K}(A)$ such that $\epsilon(a) \leq \epsilon(b)+\gamma$ and $2^{n} \gamma \leq \epsilon(b)$. Write $\gamma=\left[c_{i}\right]_{i \in \omega}$; it follows that there is $i$ in $\omega$ such that $a \leq_{n+1} b+c_{i}$ and $2^{n} c_{i} \leq_{0} b$. Hence ([15], ch. 6) there are $u \ll_{n} b$ and $v \ll_{n} c_{i}$ such that $a \leq b+c_{i}+u+v$. If $c=c_{i}+u+v$, it follows that $c \ll_{n-2} b$ and $a \leq b+c$, which concludes the proof.

As an immediate corollary, we see immediately that the notion of strongly reduced product is a generalization of the notion $\mathrm{K}(A)$ :

Corollary 3.2. Let $A$ be a refinement P.O.M.. Then $\mathrm{K}(A)$ is exactly the strongly reduced power of $A$ by the Fréchet filter over $\omega$.

We mention here another fact, whose proof relies heavily on 3.1.

Corollary 3.3. Any strongly reduced product of refinement P.O.M.'s relatively to an anticomplete filter is a refinement P.O.M..

Proof. Let $\left(A_{i}\right)_{i \in I}$ be a family of refinement P.O.M.'s, let $\mathcal{F}$ be an anticomplete filter over $I$; put $A=\bar{\prod}_{\mathcal{F}} A_{i}$, let $a=\left(a_{i}\right)_{i}, b=\left(b_{i}\right)_{i}, a^{\prime}=\left(a_{i}^{\prime}\right)_{i}, b^{\prime}=\left(b_{i}^{\prime}\right)_{i}$ in $A$. Using 3.1 and the anticompleteness of $\mathcal{F}$, we see easily that there are $X$ in $\mathcal{F}$ and a map $h: X \rightarrow \omega$ such that $\lim _{\mathcal{F}} h=\infty$ and

$$
(\forall i \in X)\left(a_{i}+a_{i}^{\prime} \equiv_{h(i)+1} b_{i}+b_{i}^{\prime}\right) .
$$

(We use the fact that in a refinement P.O.M., $a \leq_{n+2} b$ and $b \leq_{n+2} a$ implies $a \equiv_{n} b$ ). Thus, using results in chapter 6 of [15], for all $i$ in $X$, there are $u_{i} \ll_{h(i)} a_{i}, u_{i}^{\prime} \ll_{h(i)} a_{i}^{\prime}$, $v_{i} \ll_{h(i)} b_{i}, v_{i}^{\prime} \ll_{h(i)} b_{i}^{\prime}$ such that $\left(a_{i}+u_{i}\right)+\left(a_{i}^{\prime}+u_{i}^{\prime}\right)=\left(b_{i}+v_{i}\right)+\left(b_{i}^{\prime}+v_{i}^{\prime}\right)$ (and we put e.g. $u_{i}=v_{i}=u_{i}^{\prime}=v_{i}^{\prime}=0$ for all $i$ in $\left.I \backslash X\right)$. If $p_{i}, q_{i}, r_{i}, s_{i}$ refine (in the obvious sense) $\left(a_{i}+u_{i}, a_{i}^{\prime}+u_{i}^{\prime}\right),\left(b_{i}+v_{i}, b_{i}^{\prime}+v_{i}^{\prime}\right)$, then the corresponding elements $[p],[q],[r],[s]$ of $A$ refine $\left([a]+[u],\left[a^{\prime}\right]+\left[u^{\prime}\right]\right),\left([b]+[v],\left[b^{\prime}\right]+\left[v^{\prime}\right]\right)$; but since $\lim _{\mathcal{F}} h=\infty$, we have $[a]+[u]=[a],\left[a^{\prime}\right]+\left[u^{\prime}\right]=\left[a^{\prime}\right],[b]+[v]=[b],\left[b^{\prime}\right]+\left[v^{\prime}\right]=\left[b^{\prime}\right]$. So we have proved that 
$A$ satisfies the finite refinement property; the proof of minimality of $A$ is similar (in fact simpler). The conclusion follows.

We know no direct proof of this fact without the help of 3.1; in fact, we do not even know whether any strongly reduced product of minimal P.O.M.'s is minimal, although we suspect it is not the case. Note that we could have proved a similar statement about e.g. preservation of strong refinement P.O.M.'s, but it is actually redundant - more precisely, any strongly reduced product relatively to an anticomplete filter of refinement P.O.M.'s is a strong refinement P.O.M. (and it actually satisfies many more properties). The proof of this proposition uses 2.4 and results in the last chapter of [15].

\section{4. $\delta$-COMPLETE P.O.M.'s.}

First of all, we have to mention that if $A$ is a regular P.O.M., which means that its evaluation map is an embedding, then the closure $\bar{A}$ of [the image of] $A$ in $\overline{\mathbb{P}}^{A^{*}}$ is, equipped with the canonical metric $\delta$, a complete metric space, thus the metric completion of $A$. Moreover, using 1.1 and 1.3 , it is easy to see that $\bar{A}$ is closed under addition, and if $a=\lim _{n \rightarrow \infty} a_{n}$ and $b=\lim _{n \rightarrow \infty} b_{n}$ are elements of $\bar{A}$, then $a \leq b$ if and only if $\lim _{n \rightarrow \infty} \rho\left(a_{n}, b_{n}\right)=0$. It follows easily that $\bar{A}$ is itself a P.O.M.:

The metric completion of a regular P.O.M. is a regular P.O.M..

This result, however elegant, is a priori not very useful, since it does not give convenient completeness criteria for some other known classes of P.O.M.'s which already enjoy some kind of 'completeness' (intuitively rather stronger than $\delta$-completeness), as for example [weak] cardinal algebras, or even the $\mathrm{K}(A)$ 's mentioned in previous paragraph. We shall answer this question by giving a certain class of regular refinement P.O.M.'s, including both classes above, and enjoying $\delta$-completeness.

First, we need a lemma, which we already proved in [15], chapter 1.

Lemma 4.1. Let $A$ be a refinement P.O.M., let $m$ in $\mathbb{N} \backslash\{0\}$, let $a, b, c$ in $A$. Then the following are equivalent:

(i) $a+b=m c$;

(ii) There are $x_{k}(k \leq m)$ in $A$ such that

$$
\left\{\begin{array}{l}
a=\sum_{k \leq m} k x_{k} \\
b=\sum_{k \leq m}(m-k) x_{k} \\
c=\sum_{k \leq m} x_{k}
\end{array}\right.
$$


Lemmas 4.2 to 4.6 will now lead us to our solution.

Lemma 4.2. ("Parallelogram lemma") Let $A$ be a refinement P.O.M. satisfying the multiplicative $2-\leq$-cancellation property, let $n$ in $\mathbb{N}$, let $a, b$ in $A$ such that $a \leq_{n} b$. Then there are $c, a^{\prime}, d$ in $A$ such that:

(i) $a \leq b+c$ and $2^{n} c \leq b$;

(ii) $a=a^{\prime}+d$ and $2^{n} d \leq a^{\prime} \leq b$ and $d \leq c$.

The picture is as follows (an arrowed line from $x$ to $y$ means that $x \leq y$ ):

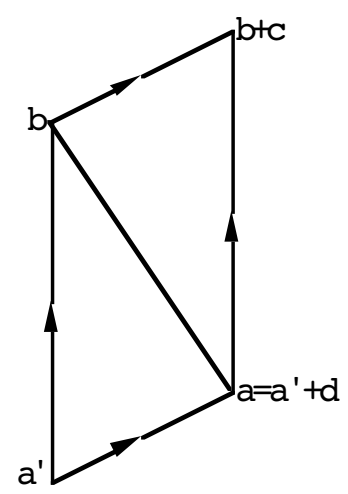

Proof. By definition of $\leq_{n}$, there is $c$ in $A$ such that $a \leq b+c$ and $c \ll_{n} b$; since $A$ satisfies the multiplicative 2 - $\leq$-cancellation property, we have $2^{n} c \leq b$, so (i) follows. Since $A$ is minimal, there is $e$ in $A$ such that $2^{n} c+e=b$, so that $a \leq\left(2^{n}+1\right) c+e$; since $A$ is a refinement P.O.M., there are $u, v$ such that $a=u+v$ and $u \leq\left(2^{n}+1\right) c$ and $v \leq e$. By 4.1 , there are $x_{k}\left(k \leq 2^{n}+1\right)$ such that $u=\sum_{k \leq 2^{n}+1} k x_{k}$ and $c=\sum_{k \leq 2^{n}+1} x_{k}$. Let $d=x_{2^{n}+1}$ and $a^{\prime}=\sum_{k \leq 2^{n}} k x_{k}+2^{n} x_{2^{n}+1}+v$. Then $a^{\prime}$, $d$ satisfy the required conditions.

Lemma 4.3. Let $A$ be an Archimedean strong refinement P.O.M. satisfying the $\left(2, \aleph_{0}\right)$ interpolation property. Then $A$ is a regular P.O.M..

Proof. We start with a

Claim. A satisfies the multiplicative 2 -- -cancellation property.

Proof of claim. An adapted version of the proof of [12], 2.31 (see also [15], chapter 3). We put $T=\{(a, b, c) \in A \times A \times A: 2 a+c \leq 2 b+c\}$. If $(a, b, c)$ is a fixed element of $T$, we get, using the fact that $A$ is a refinement P.O.M., a sequence $\left(\left(a_{n}, b_{n}, c_{n}\right)\right)_{n \in \omega}$ of elements of $T$ such that $a_{0}=a, b_{0}=b, c_{0}=c$ and for all $n$,

$$
a_{n}+c_{n}=2 a_{n+1}+c_{n+1} \text { and } b_{n}+c_{n}=a_{n+1}+b_{n+1}+c_{n+1} \text {. }
$$


Thus, for all $n$, we have $a+c \leq b+c+a_{n+1}$; it follows by the $\left(2, \aleph_{0}\right)$-interpolation property that there is $e$ in $A$ such that

$$
\{a+c, b+c\} \leq e \leq\left\{b+c+a_{n+1}: n \in \omega\right\}
$$

Let $d$ such that $e=(b+c)+d$. But for all $n$, we have $b+c \geq \sum_{i<n} a_{i+1}$, so it follows from $(2)$ that $2(b+c) \geq n d+(b+c)$, thus, using the pseudo-cancellation property, $n d \leq b+c$. Since $A$ is Archimedean, it follows that $d \ll b+c$, so that $a+c \leq b+c+d=b+c$.

Since $2 a \leq 2 b$ means that $(a, b, 0) \in T$, we get the result.

【laim.

Now, we can prove our lemma. For that purpose, it is very convenient to use the 'second embedding theorem' of [15] (whose version stated here is lemma 3.1). We first have to check that $\leq$ is antisymmetric, which results immediately from the minimality and Archimedean property of $A$. Now, let $a, b$ in $A$ such that for all $n$, we have $a \leq_{n} b$. By the claim, there is a sequence $\left(c_{n}\right)_{n}$ such that for all $n$, we have $2^{n} c_{n} \leq b$ and $a \leq b+c_{n}$. Using again $\left(2, \aleph_{0}\right)$-interpolation, there is $d$ such that $\{a, b\} \leq d \leq\left\{b+c_{n}: n \in \omega\right\}$. Let $c$ such that $b+c=d$. For all $n$, we have $b+2^{n} c \leq b+2^{n} c_{n} \leq 2 b$, thus, using the pseudocancellation property, $2^{n} c \leq b$. Since $A$ is Archimedean, we get $c \ll b$; hence $a \leq b+c=b$. The conclusion follows.

Remark. The same conclusion could have been reached (with about the same proof) if we had replaced among the assumptions about $A$ the pseudo-cancellation property by the following weaker assumption:

$$
(\forall a, b)(a \ll 2 b \Rightarrow a \ll b)
$$

(which itself results from the multiplicative 2 -=-cancellation property).

Now, from 4.4 to 4.6 , we fix a strong refinement P.O.M. $A$ satisfying the $\aleph_{0}$-absorption property, the $\left(\aleph_{0}, \aleph_{0}\right)$-interpolation property and the multiplicative $\leq$-cancellation property.

Lemma 4.4. Let $a, b, c_{i}(i \in \omega)$ in $A$ such that $(\forall i \in \omega)\left(a+c_{i} \leq b\right)$. Then there is $c$ in $A$ such that $a+c \leq b$ and $(\forall i \in \omega)\left(c_{i} \leq c\right)$.

Proof. (Note the similarity with 2.25 of [12]). Since $a \leq b$, there is $d$ such that $a+d=b$. For all $i$ in $\omega, a+c_{i} \leq b=a+d$, thus, by the pseudo-cancellation property, there is $e_{i} \ll a$ such that $c_{i} \leq d+e_{i}$. By the $\aleph_{0}$-absorption property, there is $e \ll a$ such that $(\forall i \in \omega)\left(e_{i} \leq e\right)$. Then $c=d+e$ satisfies the required condition. 
Lemma 4.5. Let $m$ in $\mathbb{N} \backslash\{0\}$, let $a, b, c_{i}(i \in \omega)$ in $A$ such that $(\forall i \in \omega)\left(m c_{i}+a \leq b\right)$. Then there is $c$ in $A$ such that $(\forall i \in \omega)\left(c_{i} \leq c\right)$ and $m c+a \leq b$.

Proof. As in [12], 2.39: the assumptions made on $A$ here are sufficient to make the proof work.

We turn now to a sort of infinite version of the 'parallelogram lemma' 4.2.

Lemma 4.6. Let $a, a_{k}(k \in \omega)$ in $A$, let $n$ in $\mathbb{N}$. Then the following holds:

(i) If $(\forall k \in \omega)\left(a_{k} \leq_{n} a\right)$, then there is $c$ in $A$ such that $2^{n} c \leq a$ and $(\forall k \in \omega)\left(a_{k} \leq a+c\right)$;

(ii) If $(\forall k \in \omega)\left(a \leq_{n} a_{k}\right)$, then there are $a^{\prime}, d$ in $A$ such that $a=a^{\prime}+d$ and $2^{n} d \leq a^{\prime}$ and $(\forall k \in \omega)\left(a^{\prime} \leq a_{k}\right)$.

Proof. Assume first the hypotheses of (i). By the $2-\leq$-cancellation property, for all $k$ in $\omega$, there is $c_{k}$ in $A$ such that $a_{k} \leq a+c_{k}$ and $2^{n} c_{k} \leq a$. By 4.5, there is $c$ such that $(\forall k \in \omega)\left(c_{k} \leq c\right)$ and $2^{n} c \leq a$. Then $c$ satisfies the required conditions for (i).

Assume now the hypotheses of (ii). By 4.2, for all $k$ in $\omega$, there are $a_{k}^{\prime}, d_{k}$ such that $a=a_{k}^{\prime}+d_{k}$ and $2^{n} d_{k} \leq a_{k}^{\prime} \leq a_{k}$. Thus $\left(2^{n}+1\right) d_{k} \leq a$, thus, by 4.5 , there is $d$ in $A$ such that $\left(2^{n}+1\right) d \leq a$ and $(\forall k \in \omega)\left(d_{k} \leq d\right)$. Thus, there is $a^{\prime} \geq 2^{n} d$ such that $a^{\prime}+d=a$. Hence, for all $k$ in $\omega$, we have $a^{\prime}+d_{k} \leq a^{\prime}+d=a=a_{k}^{\prime}+d_{k}$, thus, by the pseudo-cancellation property, $a^{\prime} \leq a_{k}^{\prime}+e_{k}$ for some $e_{k} \ll d_{k}$; but $d_{k} \leq 2^{n} d_{k} \leq a_{k}^{\prime}$, thus $e_{k} \ll a_{k}^{\prime}$; it follows that in fact, $a^{\prime} \leq a_{k}^{\prime}$. Hence, $a^{\prime}$ and $d$ satisfy the required conditions for (ii).

We can now state our theorem (see the introduction for the terminology used):

TheOREM 4.7. Let $A$ be an Archimedean strong refinement P.O.M. satisfying the $\aleph_{0}$ absorption property and the $\left(\aleph_{0}, \aleph_{0}\right)$-interpolation property. Then $A$ is a regular P.O.M., and, equipped with its canonical distance, is a complete metric space.

Proof. First, 4.3 implies that $A$ is regular. Let $\left(a_{n}\right)_{n}$ be a Cauchy sequence in $A$; to prove that it is convergent, we can without loss of generality replace it by one of its subsequences, and thus assume, using 3.1, that

$$
(\forall n \in \omega)(\forall k \geq n)\left(a_{n} \equiv_{n} a_{k}\right)
$$

Let $n$ in $\omega$. Since $(\forall k \geq n)\left(a_{k} \leq_{n} a_{n}\right)$, there is, by 4.6, $c_{n}$ in $A$ such that

$$
2^{n} c_{n} \leq a \text { and }(\forall k \geq n)\left(a_{k} \leq a_{n}+c_{n}\right)
$$


Since $(\forall k \geq n)\left(a_{n} \leq_{n} a_{k}\right)$, there are, by $4.6, a_{n}^{\prime}$ and $d_{n}$ such that

$$
a_{n}=a_{n}^{\prime}+d_{n} \text { and } 2^{n} d_{n} \leq a_{n}^{\prime} \text { and }(\forall k \geq n)\left(a_{n}^{\prime} \leq a_{k}\right)
$$

Now, let $p, q$ in $\omega$, let $k \geq p, q$; by (1) and (2), we have $a_{p}^{\prime} \leq a_{k} \leq a_{q}+c_{q}$, thus $a_{p}^{\prime} \leq a_{q}+c_{q}$. By the $\left(\aleph_{0}, \aleph_{0}\right)$-interpolation property, there is $a$ in $A$ such that

$$
(\forall p, q \in \omega)\left(a_{p}^{\prime} \leq a \leq a_{q}+c_{q}\right)
$$

It follows immediately that for all $n$ in $\omega$, we have $a_{n} \leq_{n} a$ and $a \leq_{n} a_{n}$, which shows, using 3.1 , that $a$ is the limit of $\left(a_{n}\right)_{n}$.

We can now give four important classes of $\delta$-complete regular P.O.M.'s:

Example 2. Positive cones of directed abelian ordered groups. An immediate application of theorem 4.7 yields the following

Proposition 4.8. Let $A$ be a directed abelian ordered group satisfying the $\left(\aleph_{0}, \aleph_{0}\right)$ interpolation property and the statement

$$
(\forall a, b \geq 0)((\forall n \in \mathbb{N})(n a \leq b) \Rightarrow a=0)
$$

Then $A$ embeds into a complete $\ell$-group and $A_{+}$is $\delta$-complete.

Proof. By 4.7, $A_{+}$is regular; thus (see [15], chapter 6) $A$ embeds into a complete $\ell$-group. Moreover, $\delta$-completeness follows immediately from 4.7 .

Note that $(*)$ is strictly weaker than the definition of the Archimedean property appearing in [1]; for example, the P.O.M. of equidecomposability types of polyhedra of $\mathbb{R}^{3}$ modulo isometries (with only polyhedral pieces allowed in the decompositions) is the positive cone of a directed abelian ordered group (by Zylev's theorem) which satisfies (*), but which is not Archimedean in the sense of [1] (see [2] for more details). Still, in the context of 4.8 , it follows from the fact that $A$ embeds into a complete $\ell$-group that both definitions of the Archimedean property are equivalent (see [15]).

Example 3. Weak cardinal algebras. If $\left(A,+, 0, \sum\right)$ is a weak cardinal algebra, then, equipped with its minimal preordering, it satisfies the hypotheses of 4.7 (see [9], [15]). Thus,

[The P.O.M. associated with] any weak cardinal algebra is $\delta$-complete. 
In fact, in any weak cardinal algebra, one can even assign a limit to every 'Cauchyincreasing' sequence, that is, a sequence $\left(a_{n}\right)_{n}$ such that $\lim _{(m, n) \rightarrow \infty, m \leq n} \rho\left(a_{m}, a_{n}\right)=0$. The relevant notion of limit shows in both following propositions:

Lemma 4.9. Let $A$ be a P.O.M. satisfying all the hypotheses of 4.7 , let $\left(a_{n}\right)_{n}$ be a sequence of elements of $A$ such that

$$
(\forall n \in \omega)(\forall k \geq n)\left(a_{n} \leq_{n} a_{k}\right) .
$$

Then there is an increasing sequence $\left(b_{n}\right)_{n}$ of elements of $A$ such that

$$
(\forall m, n \in \omega)\left(m \leq n \Rightarrow b_{m} \leq a_{n} \leq_{n} b_{n}\right)
$$

Proof. Let $a_{n}^{\prime}, d_{n}(n \in \omega)$ be defined as in the proof of 4.7. We construct inductively $\left(b_{n}\right)_{n}$ the following way. First, $b_{0}=a_{0}^{\prime}$. Suppose that $b_{i}$ have been defined for all $i \leq n$ such that $a_{i}^{\prime} \leq b_{i} \leq a_{i}$ and $(\forall k \geq i)\left(b_{i} \leq a_{k}\right)$ and $\left(b_{i}\right)_{i \leq n}$ is increasing. Then we have $\left\{b_{n}, a_{n+1}^{\prime}\right\} \leq\left\{a_{k}: k \geq n+1\right\}$, thus, using the $\left(2, \aleph_{0}\right)$-interpolation property, there is $b_{n+1}$ such that $\left\{b_{n}, a_{n+1}^{\prime}\right\} \leq b_{n+1} \leq\left\{a_{k}: k \geq n+1\right\}$. Thus $\left(b_{n}\right)_{n}$ is as desired.

Corollary 4.10. Let $A$ be a weak cardinal algebra, let $\left(a_{n}\right)_{n}$ be a Cauchy-increasing sequence of elements of $A$. Then the set of all $x$ in $A$ such that $\lim _{n \rightarrow \infty} \rho\left(a_{n}, x\right)=0$ has a least element, which is the limit of $\left(a_{n}\right)_{n}$ when $\left(a_{n}\right)_{n}$ is a $\delta$-Cauchy sequence.

Proof. Without loss of generality, we may assume that $(\forall n \in \omega)(\forall k \geq n)\left(a_{n} \leq_{n} a_{k}\right)$. Let $\left(b_{n}\right)_{n}$ as in 4.9; put $a=\bigvee_{n} b_{n}$ and $S=\left\{x \in A: \lim _{n \rightarrow \infty} \rho\left(x, a_{n}\right)=0\right\}$. For all $n$ in $\omega$, we have $a_{n} \leq_{n} b_{n} \leq a$, thus $a \in S$. Moreover, for all $x$ in $S$ and all $m, n$ in $\mathbb{N}$, there is $k \geq n$ such that $a_{k} \leq_{m} x$; but $b_{n} \leq a_{k}$, thus $b_{n} \leq_{m} x$; this holds for all $m$, thus $b_{n} \leq x$; thus $a \leq x$. This proves that $a=\bigwedge S$. Finally, if $\left(a_{n}\right)_{n}$ is a $\delta$-Cauchy sequence, let $\left(c_{n}\right)_{n}$ be as in the proof of 4.7. Then for all $n$, we have $(\forall k \geq n)\left(a_{k} \leq a_{n}+c_{n}\right)$, thus $a_{n}+c_{n} \in S$, thus $a \leq a_{n}+c_{n}$, thus $a \leq_{n} a_{n}$. Since $a_{n} \leq_{n} b_{n} \leq a$, we have $a=\lim _{n \rightarrow \infty} a_{n}$.

By the last statement of 4.10, the least element of the set $S$ above can be denoted by $\lim _{n \rightarrow \infty} a_{n}$ (since there is coherence with the notation for the limit of a Cauchy sequence). Then it is easy to see that the addition is continuous with respect to this notion of limit, which means that for all Cauchy-increasing sequences $\left(a_{n}\right)_{n}$ and $\left(b_{n}\right)_{n}$ of the reference weak cardinal algebra, the sequence $\left(a_{n}+b_{n}\right)_{n}$ is Cauchy-increasing and we have

$$
\lim _{n \rightarrow \infty}\left(a_{n}+b_{n}\right)=\lim _{n \rightarrow \infty} a_{n}+\lim _{n \rightarrow \infty} b_{n}
$$


Let us finally make a remark concerning simple cardinal algebras (see [5], [12]). It is not difficult to prove that any regular separable (with the canonical metric) P.O.M. has the ccc (i.e. it has no strictly increasing $\omega_{1}$-sequences) - note that the converse is false, see e.g. $\overline{\mathbb{P}}^{\omega}$; from that and the fact that any simple cardinal algebra with the ccc is linearly ordered, it is easy to see that any non-linearly ordered simple cardinal algebra (if it exists) is, equipped with its canonical distance, a non-separable metric space. According to 4.7 above and the results of [5], it follows that if $A$ is a non-linearly ordered simple cardinal algebra, then the set of all finite elements of $A$ is the positive cone of a complete vector lattice which is also a non-separable Fréchet space.

Example 4. Preservation of completeness under products. We give this example only in the context of regular P.O.M.'s, where it makes sense. Notice first that any product of regular P.O.M.'s is regular: this is because a P.O.M. is regular if and only if it embeds into a power of $\overline{\mathbb{P}}$.

Proposition 4.11. Any product of regular $\delta$-complete P.O.M.'s is regular $\delta$-complete.

Proof. Let $\left(E_{i}\right)_{i \in I}$ be a family of regular $\delta$-complete P.O.M.'s; we have seen that the P.O.M. $E=\prod_{i \in I} E_{i}$ is a regular P.O.M.. Now, let $\left(a^{n}\right)_{n \in \omega}$ be a $\delta$-Cauchy sequence of $E$; put $a^{n}=\left(a_{i}^{n}\right)_{i}$. Applying 1.2 to the projections from $E$ to the $E_{i}$ 's, we see that each sequence $\left(a_{i}^{n}\right)_{n \in \omega}$ is a $\delta$-Cauchy sequence of $E_{i}$, thus converges to some $a_{i}$ in $E_{i}$; let $a=\left(a_{i}\right)_{i}$, we prove that $a=\lim _{n \rightarrow \infty} a^{n}$. Let $\varepsilon>0$, let $N$ in $\mathbb{N} \backslash\{0\}$ such that $\ln (1+1 / N) \leq \varepsilon$; put $\eta=\ln (1+1 / N)$. There is $k$ in $\omega$ such that for all $m, n \geq k$, we have $\delta\left(a^{m}, a^{n}\right) \leq \eta$. Thus for all $i$ in $I, \delta\left(a_{i}^{m}, a_{i}^{n}\right) \leq \eta$ (use 1.2); making $m$ go to infinity yields that for all $n \geq k, \delta\left(a_{i}, a_{i}^{n}\right) \leq \eta$. Therefore, since $E_{i}$ is regular, $N a_{i} \leq(N+1) a_{i}^{n}$ and $N a_{i}^{n} \leq(N+1) a_{i}$; thus for all $n \geq k, \delta\left(a, a^{n}\right) \leq \eta \leq \varepsilon$, which concludes the proof.

As a corollary, in the context of 4.11 (and 1.3), any closed sub-P.O.M. of $\prod_{i} E_{i}$ is $\delta$-complete. For example,

- The sub-P.O.M. of $E$ whose elements are the elements of countable support of $\prod_{i} E_{i}$ is $\delta$-complete;

- For any P.O.M. $A$ and any $\delta$-complete P.O.M. $E$, $\operatorname{Hom}(A, E)$ is $\delta$-complete; for example, if $E$ is a weak cardinal algebra, then $\operatorname{Hom}(A, E)$ is always $\delta$-complete. This is interesting, because $\operatorname{Hom}(A, E)$ is not always itself a weak cardinal algebra - it may not even satisfy the finite refinement $\operatorname{property}($ see $[15])$.

Example 5. Strongly reduced products of P.O.M.'s with respect to an anticomplete 
filter. Recall that a filter is anticomplete when some sequence of its elements has empty intersection.

Proposition 4.12. Let $\left(A_{i}\right)_{i \in I}$ be a family of P.O.M.'s, let $\mathcal{F}$ be an anticomplete filter on $I$. Then $\bar{\prod}_{\mathcal{F}} A_{i}$ is $\delta$-complete.

Proof. A diagonal argument (using theorem 2.1), similar to (and in fact easier than) the one in the proof of 2.4 .

Note again that in the case where $\mathcal{F}$ is not anticomplete, $\bar{\prod}_{\mathcal{F}} \mathbb{Q}_{+}$is not $\delta$-complete.

Note also that in fact, any strongly reduced product of refinement P.O.M.'s satisfies the hypotheses of theorem 4.7 (this can be proved using 2.4 and results in [15]). This suggests that the hypotheses of 4.7 are in fact much stronger than $\delta$-completeness, and serve some other 'algebraic' property which implies $\delta$-completeness; we suspect this (hypothetic) algebraic property to be a homomorphism extension property (see also [10], [15] for the study of some examples of this kind of property). 


\section{BIBLIOGRAPHY}

[1] G. BIRKHOFF, "Lattice Theory", American Mathematical Society, Providence, Rhode Island, 1961.

[2] W.G. BOLTIANSKII, "Hilbert's third problem", translated by R. Silverman, Washington, D.C.: Winston, 1978.

[3] C.C. CHANG and H.J. KEISLER, "Model Theory", North Holland Publishing Company, 1973.

[4] R. CHUAQUI, "Cardinal algebras of functions and integration", Fundamenta Mathematicae 71, 1971, pp. 77-84.

[5] R. CHUAQUI, "Simple cardinal algebras and their applications to invariant measures", Notas Mathemáticas, Universidad Católica de Chile, vol. 6, pp. 106-131, 1976.

[6] M. FOREMAN and WEHRUNG, F., "The Hahn-Banach theorem implies the existence of a non-measurable set", to appear in Fundamenta Mathematicae.

[ ] J. KEISLER, see [3].

[7] S. KOPPELBERG, "General theory of Boolean algebras", in "Handbook of Boolean algebras", vol. 1, pp. 1-307, edited by J.D. Monk with R. Bonnet, Elsevier, Amsterdam, 1989.

[8] K.P.S. Bhaskara RAO and SHORTT, R.M., "The dual of a refinement algebra", Lecture Notes in pure and applied mathematics, General Topology and Applications, 5th North East Conference (Marcel Dekker), vol. 134, pp. 335-367, 1991.

[9] K.P.S. Bhaskara RAO and SHORTT, R.M., "Weak cardinal algebras", preprint.

[10] K.P.S. Bhaskara RAO and SHORTT, R.M., "Extensions of semigroup valued, finitely additive measures", preprint.

[11] R.M. SHORTT, "Duality for cardinal algebras", Forum Mathematicum 2, pp. 433-450.

[ ] R.M. SHORTT, see [8], [9] and [10].

[12] A. TARSKI, "Cardinal algebras", New York: Oxford, 1949.

[13] S. WAGON, "The Banach-Tarski paradox", Cambridge University Press, 1984.

[14] F. WEHRUNG, "Théorème de Hahn-Banach et paradoxes continus et discrets", Comptes Rendus de l'Académie des Sciences, t. 310, série I, pp. 303-306, 1990.

[15] F. WEHRUNG, "Embedding into injective ordered monoids", preprint.

[ ] F. WEHRUNG, see [6]. 